\title{
Nutrición en las enfermedades inflamatorias del intestino. Una revisión
}

\author{
Nutrition in inflammatory bowel disease. A review \\ Nutrição em doenças inflamatórias intestinais. Uma revisão
}

Eduardo Moreira' ${ }^{1}$ Ximena Rodríguez², Patricia López³, Lourdes Silva ${ }^{4}$, Estela Olano5

\section{Resumen}

Existe evidencia epidemiológica en humanos, a partir de estudios de cohortes de grandes poblaciones, de que la dieta ejerce una fuerte influencia en el desarrollo y curso de la enfermedad inflamatoria intestinal (EII). Además, la mayoría de los tratamientos médicos basados en la evidencia para las Ell están dirigidos a suprimir la respuesta inmunitaria y conllevan riesgos de efectos secundarios importantes. La evidencia actual ha demostrado varios factores dietéticos que probablemente protejan contra los brotes, como la fibra, el zinc y la vitamina D en ambas formas de Ell y una alta proporción de Ácidos grasos poliinsaturados (AGPI) $n-3 / n-6$ en la dieta en la CU. Otros factores aumentan el riesgo de brotes, son los alimentos que contienen ácido mirístico, las carnes rojas y el azúcar. Esta revisión explorará las interacciones de la dieta y el sistema inmunológico en el contexto de la enfermedad de Crohn (EC) y la colitis ulcerosa (CU), centrándose en el papel de la dieta en la patogénesis, en particular la interacción con el sistema inmunológico del paciente. Al mismo tiempo se analizará el rol de la nutrición médica como intervención terapéutica.

Palabras clave: Enfermedades inflamatorias del intestino

Estado nutricional

Dietoterapia

Key words: Inflammatory bowel diseases

Nutritional status

Diet therapy

\footnotetext{
1. Médico Intensivista. Terapia Nutricional. Unidad de Nutrición e Insuficiencia Intestinal (UNNII) del Hospital Maciel. ASSE, Montevideo, Uruguay. 2. Gastroenteróloga. Ex - Asistente de la Clínica de Gastroenterología. Hospital de Clínicas, Facultad de Medicina. UdelaR. Montevideo, Uruguay. Unidad de Enfermedades Inflamatorias Intestinales del Hospital Maciel. ASSE, Montevideo, Uruguay.

3. Cirujana. Profesora Adjunta de Clínica Quirúrgica. Hospital de Clínicas, Facultad de Medicina. UdelaR. Montevideo, Uruguay.

4. Licenciada en enfermería. Terapia Nutricional. Unidad de Nutrición e Insuficiencia Intestinal (UNNII) del Hospital Maciel. ASSE, Montevideo, Uruguay.

5. Médico Intensivista. Terapia Nutricional. Coordinadora de la Unidad de Nutrición e Insuficiencia Intestinal (UNNII) del Hospital Maciel. ASSE, Montevideo, Uruguay.

Los autores declaran no tener conflicto de intereses.

Todos los autores participaron de igual forma en la elaboración del presente manuscrito.

Correspondencia: Dr. Eduardo Moreira. Hospital Maciel. 25 de mayo 174. Montevideo, Uruguay.

Correo electrónico: dreduardo.moreira@gmail.com

Recibido: 10/3/2021

Aprobado: 16/6/2021

Attribution-NonCommercial 4.0 International (CC BY-NC 4.0)
} 


\section{Introducción}

Las enfermedades inflamatorias del intestino (EII), la colitis ulcerosa (CU) y la enfermedad de Crohn (EC) se caracterizan por una inflamación crónica recidivante y remitente del tracto gastrointestinal. La CU se limita a la capa mucosa del colon, casi siempre involucra el recto y puede extenderse para involucrar porciones más proximales del intestino grueso de manera continua. La EC por su parte se caracteriza por determinar inflamación transmural y omisión de áreas de compromiso (segmentos de intestino de apariencia normal interrumpidos por áreas de enfermedad) de todo el tracto gastrointestinal desde la boca hasta el ano. Como resultado del compromiso del tracto gastrointestinal, la desnutrición es un problema frecuente en pacientes con EII, algunos estudios han informado una prevalencia de $70 \%-80 \%$ de los pacientes hospitalizados con EII y en $20 \%-40 \%$ de los pacientes ambulatorios con $\mathrm{EC}^{(1)}$. La desnutrición puede ocurrir tanto en la CU como en la EC, pero la prevalencia de desnutrición proteicoenergética y de nutrientes específicos parece ser mayor en la EC en comparación con la $\mathrm{CU}$, probablemente porque puede afectar cualquier parte del tracto gastrointestinal. Generalmente es secundaria a reducciones en la ingesta oral, pérdidas gastrointestinales aceleradas, al tratamiento farmacológico (glucocorticoides, sulfasalazina) y al hipermetabolismo que determina mayores requerimientos nutricionales durante la enfermedad activa. La desnutrición es uno de los factores más importantes asociados con una mala evolución clínica en pacientes con EII, por lo que la evaluación del estado nutricional y la necesidad de terapia nutricional desempeñan un papel fundamental en la atención clínica de estos pacientes ${ }^{(1)}$. En la figura 1 se resumen los mecanismos de desnutrición en pacientes con EII.

En la base etiopatogénica de las EII existe una interacción compleja, y no completamente comprendida, de múltiples factores que incluyen la genética, el sistema inmunológico, los factores microbianos y ambientales, en particular la dieta. Se han identificado mutaciones en uno o más de aproximadamente 200 genes que codifican o modulan la expresión de proteínas que afectan las funciones de regulación inmunológica en pacientes diagnosticados con EII ${ }^{(2)}$. Por otro lado, los estudios epidemiológicos revelan una mayor prevalencia de EII en países desarrollados, las tasas de incidencia se están acelerando en países occidentalizados siguiendo un patrón evidenciado previamente en el siglo XX en el mundo oc$\operatorname{cidental}^{(3)}$. Esto pone de relieve el impacto limitado de la genética y la importancia potencial de los factores ambientales en la patogénesis, sugiriendo que el estilo de vida occidental típico, incluida la dieta, puede contribuir al desarrollo de la EII. Cuando el individuo se expone a un desencadenante ambiental, puede generarse una respuesta desregulada del sistema inmunológico de la mucosa a la microbiota que reside dentro de la luz intestinal, culminando en una respuesta inflamatoria exagera$\mathrm{da}^{(4)}$. Existe un interés creciente por comprender el papel que puede desempeñar la dieta en la patogenia y el tratamiento de la EII, lo que podría traducirse en una mejora de los resultados cínicos en pacientes con EII ${ }^{(1)}$.

\subsection{Ell e inflamación}

En la EC la inflamación puede ocurrir a través de la longitud del tracto GI y no se limita a la mucosa, sino que es transmural, afectando el grosor total del tejido. La ubicación anatómica de la enfermedad puede cambiar con el tiempo, pero es más común encontrarla en el intestino delgado distal, donde se encuentra en el $47 \%$ de los casos en el momento del diagnóstico, mientras que afecta al colon en el $28 \%$ de los casos y en el $21 \%$ de los casos compromete al íleon y al colon. Por otro lado, la CU está confinada al colon y la inflamación afecta solo a la mucosa ${ }^{(5)}$.

Las EII y sus síntomas asociados se pueden atribuir a la respuesta inflamatoria prolongada y disfuncional. Los cambios en la arquitectura de la mucosa intestinal, estenosis, engrosamiento intestinal, ulceraciones, alteración de la motilidad y malabsorción son algunos ejemplos de las consecuencias de la respuesta inflamatoria intensificada en las EII. Los síntomas típicos de la enfermedad tales como dolor intestinal y extraintestinal, distensión abdominal, diarrea, esteatorrea, pérdida de peso y desnutrición son manifestaciones de niveles aumentados de citocinas locales y circulantes y otros productos de la inflamación. La EII es habitualmente una enfermedad intermitente que se caracteriza por recaídas (brotes) y remisiones. Los factores ambientales pueden desencadenar una recaída o ayudar a mantener la remisión, por lo que el conocimiento de estos factores es importante para el manejo clínico de estos pacientes. Sin embargo, los factores desencadenantes potenciales son múltiples (genéticos y ambientales), y con frecuencia son difíciles de predecir. Además, pueden transcurrir varios años entre el inicio, el daño histológico, los síntomas y el diagnóstico de EII. De particular interés es la interacción compleja entre la dieta de un paciente, su microbioma intestinal y el sistema inmunológico y cómo pueden manipularse para controlar mejor su enfermedad, mejorar la eficacia de los tratamientos y reducir los riesgos y efectos secundarios asociados con las terapias médicas tradicionales ${ }^{(6)}$. Las reacciones normales a la dieta pueden causar síntomas gastrointestinales (gases, distensión abdominal, dolor, calambres y diarrea) y pueden confundirse 


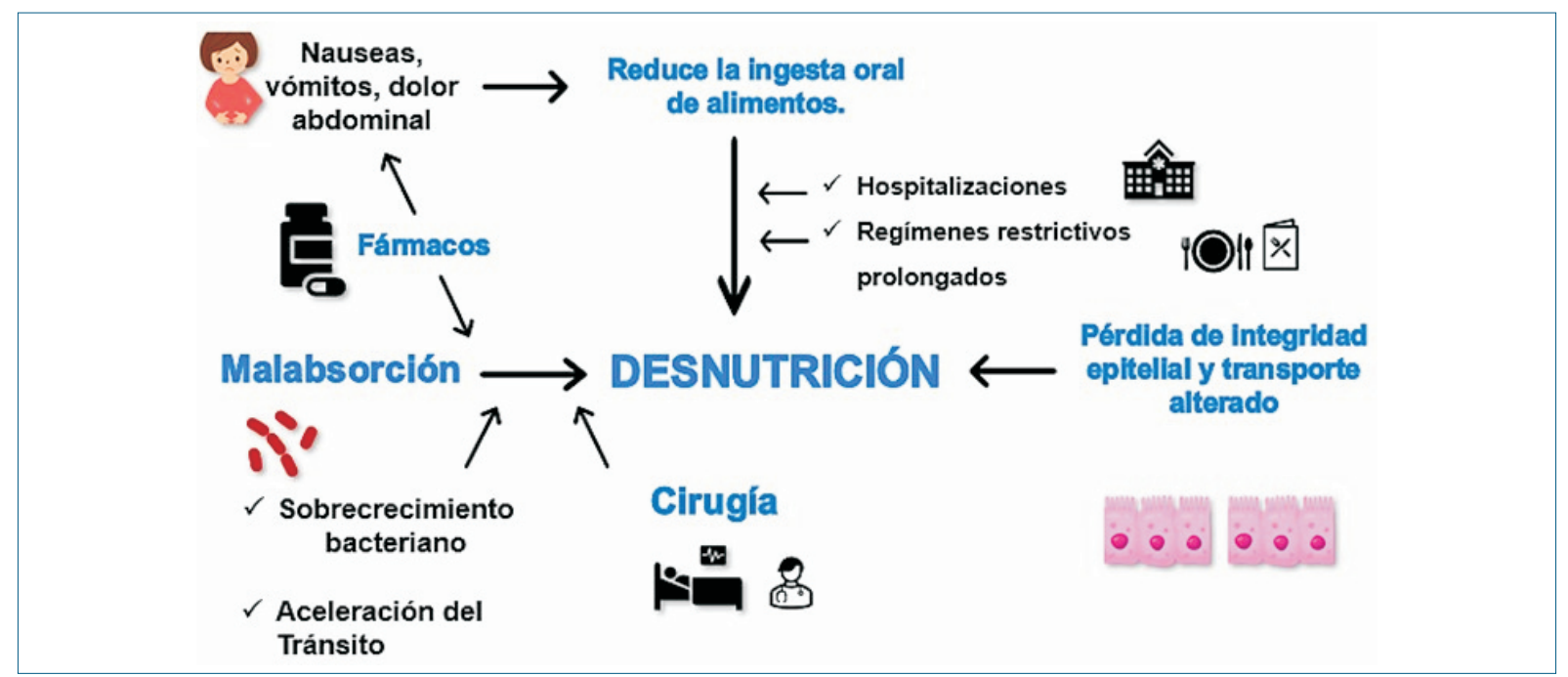

Figura 1. Mecanismos de desnutrición en pacientes con enfermedad inflamatoria intestinal.

con una enfermedad activa. Los factores dietéticos que se considera que provocan una enfermedad activa incluyen una ingesta elevada de azúcar, lípidos específicos, metabolismo anormal de aminoácidos que contienen azufre como en la hiperhomocisteinemia, ingesta inadecuada de nutrientes como el folato y vitamina $\mathrm{D}$, e intolerancias o alergias alimentarias individuales. Por otro lado, existe datos que apoyan el hecho de que la lactancia y el consumo de frutas, verduras, fibra dietética y lípidos omega-3 son protectores ${ }^{(7)}$. Si bien durante mucho tiempo ha existido la percepción del paciente sobre el papel que desempeña la dieta en el inicio y el curso de la enfermedad, la evidencia que respalda esto y cómo manipular de manera óptima la ingesta dietética y proporcionar consejos dietéticos prácticos y sólidos ha sido limitada.

\section{Aspectos nutricionales en las Ell}

Los pacientes con EII ya están en riesgo de deficiencias de micronutrientes y macronutrientes y esto puede verse agravado por la evitación de alimentos sin supervisión. Debido a la ingesta crónica deficiente, el aumento de las tasas de renovación de proteínas y el aumento de las pérdidas intestinales de nutrientes durante las fases de la enfermedad activa, puede haber una pérdida neta de proteínas. Una revisión sistemática informó que hasta un $60 \%$ de los pacientes con EII tienen una masa muscular disminuida en comparación con los sujetos sanos. ${ }^{8}$ La sarcopenia se ha asociado con una mayor necesidad de cirugía y resultados quirúrgicos deficientes en la EII, así como con la osteopenia ${ }^{(9)}$. En la tabla 1 se muestra la frecuencia de las deficiencias de micronutrientes más comunes en pacientes con EII. La deficiencia de micronutrientes esenciales conduce a efectos se-
Tabla 1. Frecuencia de deficiencias nutricionales en pacientes con enfermedad inflamatoria intestinal (ElI). (Adaptado de Balestrieri et al(47)).

\begin{tabular}{|lcc|}
\hline Déficit nutricional & \multicolumn{2}{c|}{ Enfermedad inflamatoria intestinal } \\
\cline { 2 - 3 } & $\begin{array}{c}\text { Enfermedad de Crohn } \\
(E C)\end{array}$ & $\begin{array}{c}\text { Colitis ulcerosa ( } \\
\text { CU) }\end{array}$ \\
\hline Pérdida de peso & $65 \%-75 \%$ & $18 \%-62 \%$ \\
Anemia & $60 \%-80 \%$ & $66 \%$ \\
Hierro & $39 \%$ & $81 \%$ \\
Magnesio & $14 \%-33 \%$ & S/D \\
Ácido fólico & $54 \%$ & $36 \%$ \\
Calcio & $13 \%$ & S/D \\
Vitamina D & $75 \%$ & S/D \\
Vitamina B12 & $48 \%$ & $5 \%$ \\
Vitamina K & S/D & S/D \\
\hline S/D: sin datos. & & \\
\hline
\end{tabular}

cundarios deletéreos que involucran el metabolismo, la expresión génica y el estrés oxidativo ${ }^{(10)}$. En el contexto de la EII, el papel de los micronutrientes en el control genético y la antioxidación es de particular importancia. Las vitaminas A, B y E se han implicado en la regulación de las respuestas inmunitarias y la homeostasis en el intestino, inhibiendo la liberación de citocinas inflamatorias y favoreciendo la diferenciación de los linfocitos reguladores sobre los linfocitos $\mathrm{T}$ efectores proinflamatorios $^{(11)}$. En pacientes sanos, la producción de espe- 
Tabla 2. Implicaciones inmunológicas y resultados clínicos asociados con las deficiencias de micronutrientes en la Ell. (Adaptado de Kilby y colaboradores $\left.{ }^{(48)}\right)$.

\begin{tabular}{|c|c|c|c|}
\hline Micronutriente y nivel en la Ell & Efecto de la deficiencia & $\begin{array}{c}\text { Supuesta función patogénica de la } \\
\text { deficiencia }\end{array}$ & Efecto de la suplementación \\
\hline Vitamina $A \downarrow$ & $S / D$ & $\begin{array}{l}\downarrow \text { diferenciación de células } T \\
\text { reguladores. } \\
\uparrow \text { diferenciación de células T efectores. } \\
\downarrow \text { migración de células T al intestino }\end{array}$ & $S / D$ \\
\hline Folato $\downarrow$ & $\begin{array}{l}\text { Anemia macrocítica } \\
\uparrow \text { Homocisteinemia }\end{array}$ & $\begin{array}{l}\downarrow \text { Supervivencia de células } T \\
\text { reguladores. } \\
\uparrow \text { Señalización proinflamatoria } \\
\uparrow \text { Estrés oxidativo }\end{array}$ & $\downarrow$ Riesgo de cáncer colorrectal \\
\hline Vitamina B12 $\downarrow\left(^{*}\right)$ & $\begin{array}{l}\text { Anemia macrocítica } \\
\uparrow \text { Homocisteinemia } \\
\text { Daño neurológico }\end{array}$ & $S / D$ & $S / D$ \\
\hline Vitamina $\mathrm{D} \downarrow$ & $\begin{array}{l}\uparrow \text { Marcadores inflamatorios. } \\
\uparrow \text { Utilización de la atención sanitaria. } \\
\uparrow \text { Actividad de la enfermedad. } \\
\uparrow \text { Riesgo de cáncer colorrectal. } \\
\downarrow \text { Calidad de vida }\end{array}$ & $\begin{array}{l}\text { 个Activación de las células T. } \\
\uparrow \text { Señalización proinflamatoria. } \\
\downarrow \text { Disminución del recambio de } \\
\text { células T. }\end{array}$ & $\begin{array}{l}\uparrow \text { Densidad mineral ósea } \\
\downarrow \text { Riesgo de cirugía }\end{array}$ \\
\hline Vitamina $\mathrm{K} \downarrow$ & $\begin{array}{l}\downarrow \text { Densidad mineral ósea. } \\
\uparrow \text { Riesgo de hemorragia. }\end{array}$ & 个 Señalización proinflamatoria & $\begin{array}{l}\leftrightarrow \text { Actividad de la enfermedad } \\
\leftrightarrow \text { Densidad mineral ósea }\end{array}$ \\
\hline Hierro $\downarrow$ & Anemia ferropénica & $S / D$ & $\begin{array}{l}\text { Resolución de la anemia } \\
\uparrow \text { Calidad de vida }\end{array}$ \\
\hline Zinc $\downarrow$ & $\begin{array}{l}\uparrow \text { Riesgo de EC. } \\
\uparrow \text { Hospitalizaciones } \\
\uparrow \text { Cirugía } \\
\uparrow \text { Complicaciones }\end{array}$ & $\begin{array}{l}\uparrow \text { Señalización proinflamatoria } \\
\uparrow \text { Estrés oxidativo }\end{array}$ & $\leftrightarrow$ Actividad de la enfermedad \\
\hline Selenio $\downarrow$ & ¿Complicaciones CV? & $\begin{array}{l}\text { 个 Señalización proinflamatoria } \\
\uparrow \text { Estrés oxidativo }\end{array}$ & $S / D$ \\
\hline
\end{tabular}

* Sobre todo en pacientes con resecciones ileales. $\downarrow$ : Reduce o disminuido, $\uparrow:$ Aumenta, $\leftrightarrow$ : Modula. EII: enfermedad inflamatoria intestinal. CV: cardiovasculares. S/D: Sin dato.

cies reactivas de oxígeno (ROS) se equilibra con la actividad de micronutrientes antioxidantes como las vitaminas A y E, así como las enzimas que requieren metales traza (zinc y selenio, por ejemplo) como cofactores. En la EII, la desregulación inmunitaria y la inflamación dan como resultado un aumento de la producción de ROS, lo que subyace aún más a la importancia de los micronutrientes antioxidantes para mitigar la patogénesis de la EII ${ }^{(12)}$. No está claro si es el aumento de la gravedad de la enfermedad el responsable de las deficiencias de micronutrientes al afectar la absorción o si por el contrario son los niveles cada vez menores de micronutrientes, que actúan como antioxidantes y reguladores inmunitarios, los responsables de la progresión de la EII. En la tabla 2 se presentan las implicaciones inmunológicas de las deficiencias de micronutrientes, en pacientes adultos con EII.

\subsection{Interacciones entre la dieta, el microbioma y el sistema inmune en las Ell}

La disbiosis es un desequilibrio en la composición del microbioma intestinal y es una característica de la EII. Esto se caracteriza por la pérdida de diversidad microbiana, particularmente de bacterias anaeróbicas favorables y productoras de ácidos grasos de cadena corta (AGCC) como Faecalibacterium prausnitzii , así como un mayor número de especies patógenas invasoras y adherentes desfavorables como Escherichia coli invasora adherente $^{(13)}$. Se postula que cuando la disbiosis y la función microbiana alterada se combinan con la función 


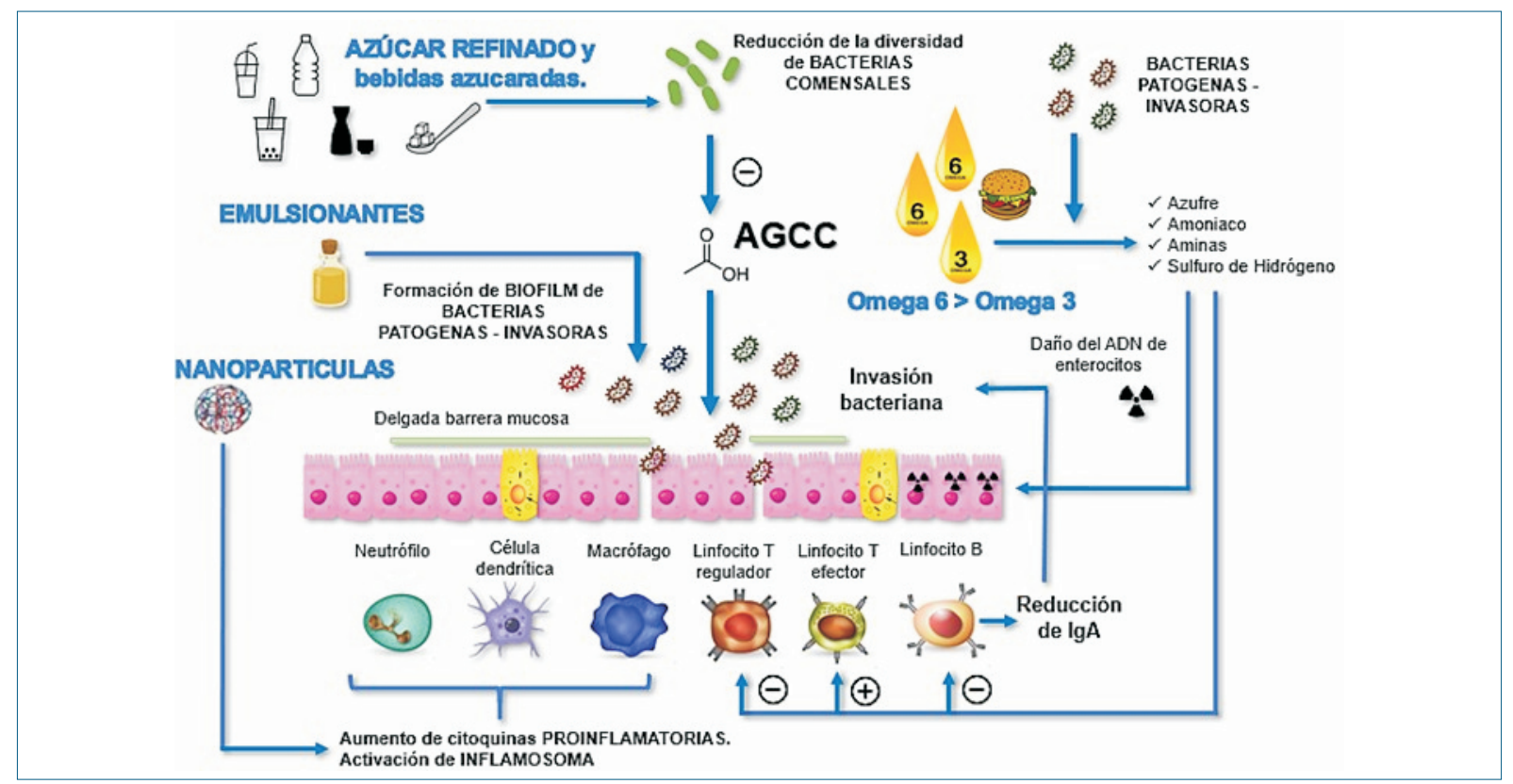

Figura 2. Interacciones entre la dieta y el sistema inmune y su impacto en las enfermedad inflamatoria intestinal (adaptado de: Wark et al ${ }^{(49)}$ ). AGCC: ácidos grasos de cadena corta.

de barrera de la mucosa alterada como ocurre en las EII, se produce inflamación de la mucosa ${ }^{(14)}$. La dieta influye en el sistema inmunológico y la respuesta inflamatoria tanto a través de su capacidad para alterar la estructura y función microbiana como a través de interacciones con las defensas de la mucosa intestinal y las células inflamatorias directamente.

En la figura 2 se esquematizan los mecanismos propuestos de interacciones patológicas entre la dieta y el sistema inmunitario que pueden contribuir al riesgo de desarrollar EII y la actividad de la enfermedad. Aunque los estudios epidemiológicos nutricionales están limitados por muchos factores de confusión, algunas relaciones fueron sólidas y consistentes entre los estudios. La ingesta de leche materna, frutas y verduras y una mayor proporción de ácidos grasos n -3/n -6 en la dieta parecen protectores mientras que la ingesta de bebidas azucaradas se asocia con un mayor riesgo de ambas formas de EII. La carne roja parece estar relacionada solo con el riesgo de $\mathrm{CU}$, y un mayor consumo de emulsionantes y grasas animales parece aumentar el riesgo de EC. La evidencia más sólida de la reducción del riesgo en el desarrollo de EC se encuentra con el pescado, particularmente como parte de una dieta mediterránea, así como con una ingesta adecuada de zinc y vitamina $\mathrm{D}$, mientras que la ingesta de ácido docosahexaenoico (DHA), un ácido graso no esencial poliinsaturado de la serie omega-3 puede reducir el riesgo de $\mathrm{CU}^{(15,16)}$. Por otro lado, la actividad de la enfermedad también puede estar modulada por la dieta, con evidencia de que la fibra dietética, una alta proporción de ácidos grasos poliinsaturados (PUFA) n-3/n -6 en la dieta puede disminuir el riesgo de brotes en ambas formas de EII. Los niveles disminuidos de zinc y vitamina $\mathrm{D}$ se han asociado inversamente con niveles de actividad de la enfermedad y peores resultados de salud en ambas formas de $\mathrm{EII}^{(17)}$. Los factores que aumentan el riesgo de brotes en la CU incluyen el ácido mirístico y la carne roja. Se ha demostrado que las dietas bajas en gluten y dietas bajas en carbohidratos de cadena corta no digeribles (FODMAP) mejoran los síntomas residuales en aquellos con EII en remisión, específicamente en aquellos con síndrome de intestino irritable concomitante ${ }^{(18)}$. En la tabla 3 se esquematiza la relación entre los componentes dietéticos y el impacto en el control de la enfermedad y en la figura 3 se muestra una representación esquemática del mecanismo propuesto de interacción favorable entre la dieta y la inmunidad intestinal que puede proteger del desarrollo de la EII y mejorar la actividad de la enfermedad.

\section{Terapia nutricional en las EEI}

Se han estudiado tres modelos o enfoques principales de intervención dietética para su uso en la práctica clínica con el objetivo de colaborar en el control de los brotes de EII fundamentalmente en la EC; estos son a) suplementación con componentes dietéticos seleccionados, b) exclusión de componentes dietéticos seleccionados y c) el uso de fórmulas dietéticas para reemplazar una dieta normal ${ }^{(19,20)}$. Debido a la naturaleza restrictiva de muchos de estos regímenes dietéticos, se reservan en 


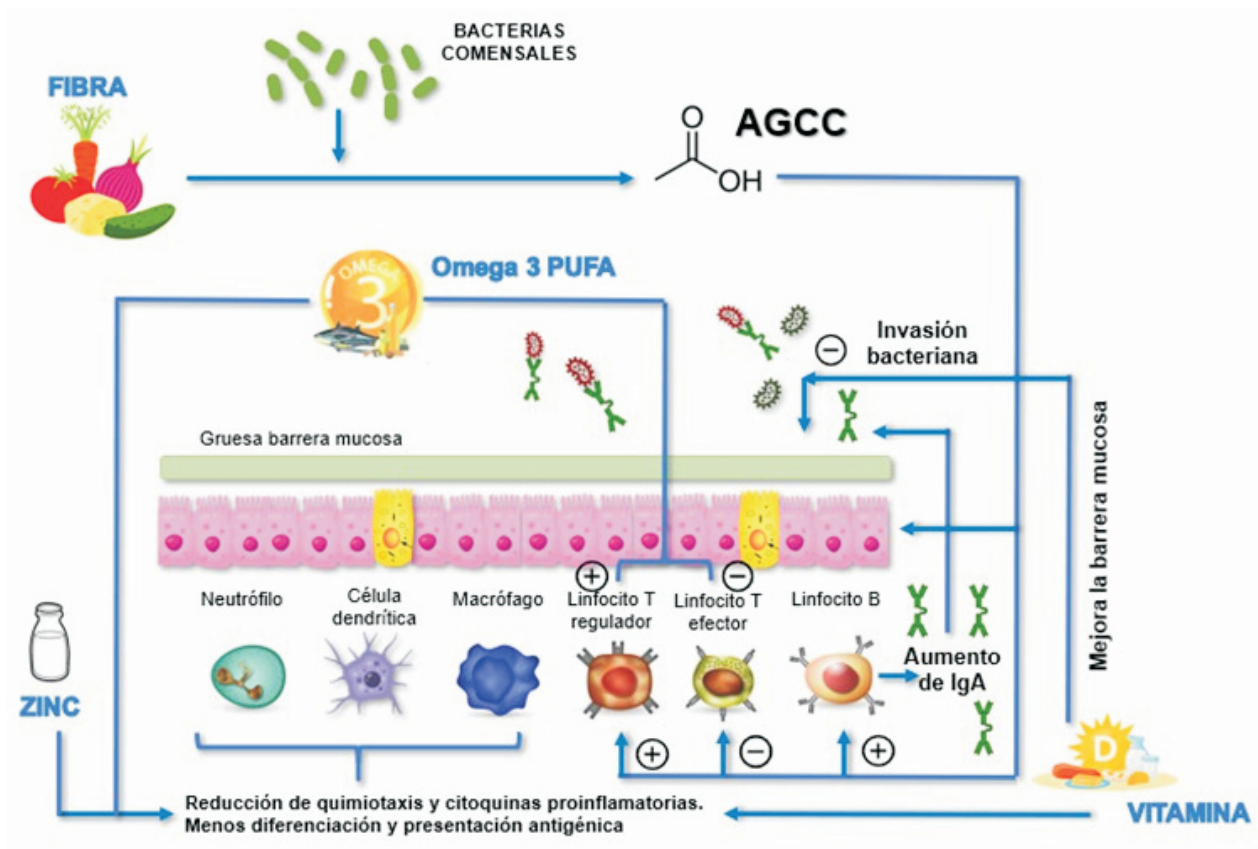

Figura 3. Interacción favorable entre la dieta e inmunidad intestinal en pacientes con enfermedad inflamatoria intestinal(adaptado de: Wark et al ${ }^{(49)}$ ). AGCC: ácidos grasos de cadena corta; PUFA: ácidos grasos poliinsaturados.

gran medida para los pacientes más graves o para aquellos que deban evitar el uso de medicamentos a base de esteroides.

\subsection{Nutrición enteral exclusiva y nutrición enteral parcial}

La nutrición enteral exclusiva (NEE) implica el suministro del $100 \%$ de las necesidades nutricionales de una persona como una fórmula nutricional líquida administrada por vía oral o enteral durante un período de 6 a 8 semanas. Se postula que la NE modula la respuesta inmune intestinal al reducir la exposición al antígeno. En consecuencia parece ejercer un efecto antiinflamatorio sobre la mucosa intestinal al reducir la producción de IL-6 y aumentar la producción de factor de crecimiento similar a la insulina (IGF) -1, generando cambios en el microbioma intestinal y el metaboloma, disminuyendo significativamente la diversidad bacteriana intestinal por debajo de los niveles previos al tratamiento y facilitando la curación de la mucosa, al limitar la actividad de la microbiota patógena con el reposo intestinal induci$\mathrm{do}^{(21)}$. En este sentido un metaanálisis y una revisión Cochrane de ensayos controlados aleatorios (ECAs) investigan el efecto de la NE en la EC ${ }^{(22,23)}$. Estos estudios han demostrado principalmente que la NE como única nutrición puede inducir la remisión clínica y la curación de la mucosa en la EC. Se ha observado que la NE es eficaz en el tratamiento de la fase aguda de la EC, con tasas de remisión que oscilan entre el $20 \%$ y el $84,2 \%$, independientemente de la ubicación de la enferme$\mathrm{dad}^{(24)}$. Otros metaanálisis más recientes han sugerido que existe beneficio de la NEE para la inducción y el mantenimiento de la remisión de la EII en comparación con el placebo en pacientes adultos con EC, pero no en comparación con los corticosteroides ${ }^{(25,26)}$.

\subsubsection{Inducción de la remisión}

El metaanálisis de Zachos y colaboradores, que incluyó 192 pacientes adultos tratados con NE y 160 con corticosteroides, indicó que la NE es menos eficaz que los corticosteroides para inducir la remisión de la EC activa. Sin embargo, el metaanálisis fue realizado hace más de diez años y los pacientes que completaron con éxito la NE lograron tasas de remisión comparables a las de los que recibieron corticosteroides ${ }^{(24)}$. Sugiriendo que una de las principales barreras para la eficacia es la adherencia del paciente al tratamiento con $\mathrm{NEE}^{(27)}$. Posteriormente un gran estudio retrospectivo chino mostró que los pacientes con EC que completaron la terapia con NEE antes del tratamiento quirúrgico de la EC tenían un riesgo menor de complicaciones y reoperaciones e intervalos libres de inmunosupresores más prolongados en comparación con los pacientes sin terapia con $\mathrm{NEE}^{(28)}$. 


\begin{tabular}{|c|c|c|}
\hline \multirow{2}{*}{$\begin{array}{l}\text { Componente } \\
\text { de la dieta }\end{array}$} & \multicolumn{2}{|c|}{ Impacto en el control de la Ell } \\
\hline & Enfermedad de Crohn & Colitis ulcerosa \\
\hline Fibra & Reduce el riesgo de brotes. & Mejora la actividad de la enfermedad. \\
\hline FODMAP & \multicolumn{2}{|l|}{ La exclusión puede mejorar los síntomas. } \\
\hline Gluten de trigo & \multicolumn{2}{|c|}{ La exclusión mejora los síntomas, especialmente en la EC estenosante. } \\
\hline Azúcar refinado & $\begin{array}{l}\text { La exclusión mejora los índices clínicos y la curación de } \\
\text { la mucosa en un pequeño estudio pediátrico. }\end{array}$ & $\begin{array}{l}\text { La exclusión generó mejoría subjetiva de los síntomas } \\
\text { informada por los pacientes, pero sin ensayos que } \\
\text { respalden. }\end{array}$ \\
\hline $\begin{array}{l}\text { Lípidos } \\
n-3 \text { PUFA versus } n-6 \text { PUFA }\end{array}$ & $\begin{array}{l}\text { Una relación más alta de PUFA n - } 3 \text { / PUFA n - } 6 \text { mejora } \\
\text { las tasas de remisión. }\end{array}$ & $\begin{array}{l}\text { Alto consumo de grasas, en particular ácido mirístico } \\
\text { (aceite de palma, el aceite de coco y la grasa láctea) } \\
\text { asociado con aumento de brotes. }\end{array}$ \\
\hline Emulsionantes & \multicolumn{2}{|l|}{ No hay estudios en humanos. } \\
\hline Nanopartículas & $\begin{array}{l}\text { Un pequeño estudio piloto mostró mejores tasas de } \\
\text { remisión, no replicado en un estudio más grande. }\end{array}$ & Sin estudios en humanos. \\
\hline Carne & Sin impacto demostrado. & La carne roja aumenta el riesgo de brotes. \\
\hline Calcio & $\begin{array}{l}\text { La dieta baja en calcio no mejoró el control de la } \\
\text { enfermedad. }\end{array}$ & $\begin{array}{l}\text { La exclusión de las proteínas de la leche de vaca no } \\
\text { mejoró el control de la enfermedad. }\end{array}$ \\
\hline Zinc & \multicolumn{2}{|c|}{ Niveles bajos de zinc asociados con un aumento de la hospitalización y requerimientos de cirugía. } \\
\hline Vitamina D & \multicolumn{2}{|c|}{$\begin{array}{l}\text { Relación inversa entre la vitamina } D \text { sérica y los índices de actividad de la enfermedad, así como peores } \\
\text { puntuaciones de calidad de vida relacionadas con la salud. }\end{array}$} \\
\hline
\end{tabular}

\subsubsection{Mantenimiento de la remisión}

La NEE podría usarse no solo para controlar el brote sino también para mantener la remisión ${ }^{(29)}$.Varios estudios han evaluado la eficacia de la NE para mantener la remisión inducida médica o quirúrgicamente ${ }^{(30,31)}$. Estos estudios han mostrado tasas de recurrencia significativamente más bajas en pacientes tratados con NEE que en aquellos con una dieta normal. Por otro lado, Takagi y colaboradores aleatorizaron a 51 pacientes con EC que habían alcanzado recientemente la remisión, 26 para recibir la mitad de su ingesta calórica como NE y 25 para tener una dieta libre ${ }^{(32)}$.Durante un seguimiento medio de 11,6 meses, la tasa de recaída fue del $34,6 \%$ en el grupo "medio enteral" y del $64 \%$ en el grupo de dieta libre. Por su parte, Hanai et al. comparó en tres grupos el efecto de la 6-mercaptopurina (6-MP), una dieta elemental y ninguna terapia en pacientes en mantenimiento de la EC. Después de 24 meses, las tasas de remisión clínica en los grupos de dieta elemental y 6-MP fueron significativamente más altas que en el grupo de control. No hubo diferencias significativas entre el grupo de 6-MP y el grupo de dieta elemental ${ }^{(33)}$. En otro estudio prospectivo que incluyó a 40 pacientes adultos con EC que alcanzaron la remisión clínica, en el que $50 \%$ de los pacientes recibieron infusión continua de dieta elemental durante la noche y una dieta baja en grasas durante el día, mientras que la otra mitad no recibió terapia nutricional ni restricción alimentaria, se observó que $25 \%$ en el grupo NE y $65 \%$ en el grupo sin NE tuvieron una recaída clínica durante el período de observación de un año ${ }^{(34)}$.

Se esperaba que la administración de nutrición enteral parcial (NEP: $50 \%$ de nutrición enteral y 50\% de dieta sin restricciones) pudiera mejorar el cumplimiento. Cuando se comparó la NEP con la NEE, el régimen de NEE fue muy superior ${ }^{(35)}$. Con la intención de optimizar el enfoque de la NEP, Levine y colaboradores diseñaron recientemente un estudio de 12 semanas que compara la NEE estándar y una dieta de exclusión de EC específica (DEEC) más la NEP en una población pediátrica ${ }^{(36)}$. Esta dieta consistió en alimentos que tenían menos probabilidades de tener efectos adversos sobre el microbioma y la barrera intestinal, evitaron los alimentos ricos en emulsionantes y edulcorantes artificiales. La tasa de aceptabilidad del paciente fue mayor con DEEC que con 


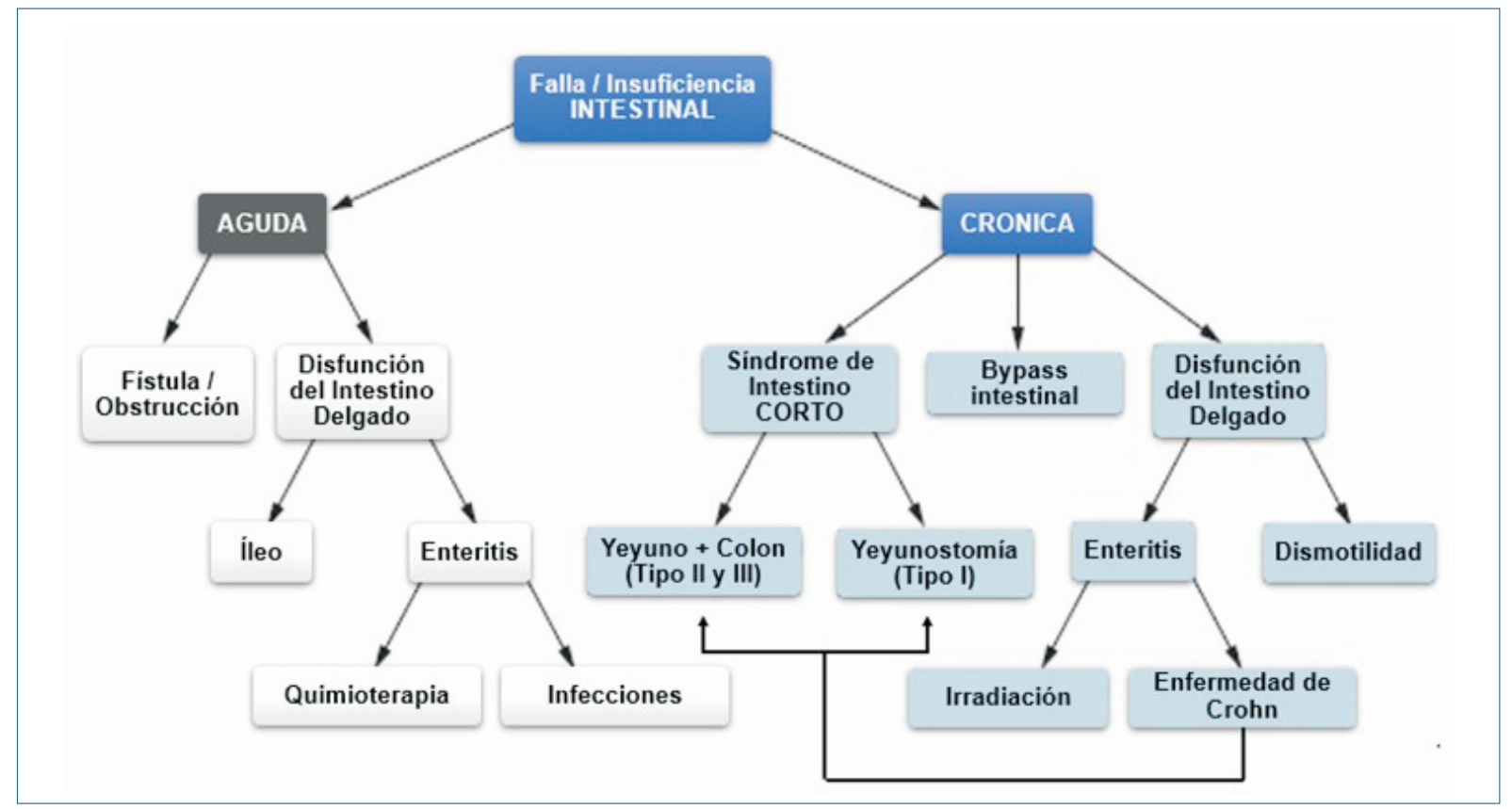

Figura 4. Causas de insuficiencia y falla intestinal en pacientes adultos.

la NEE y las tasas de respuesta clínica no fueron estadísticamente diferentes entre los dos regímenes de dieta. Esto parece prometedor, postulando la utilización de un enfoque dietético menos restrictivo para lograr tasas de remisión no inferiores a la terapia NEE. En este sentido, el estudio CD-TREAT evaluó los efectos de una dieta individualizada, cuyo objetivo era recrear los beneficios de una NEE mediante la exclusión de componentes dietéticos específicos, como el gluten, la lactosa y el alcohol. Se observó un impacto similar a la NEE en el microbioma intestinal, la inflamación y la respuesta clínica en un modelo de rata, así como en adultos y niños con EC. Esta dieta fue más fácil de cumplir para los pacientes y más aceptable que la $\mathrm{NEE}^{(37)}$.

\subsubsection{Tratamiento de la falla o insuficiencia intestinal}

La EC puede determinar una insuficiencia o falla intestinal, y esta entidad es la afección intestinal más representativa en la que la terapia nutricional constituye un componente fundamental del tratamiento en estos pacientes $^{(38)}$. En la figura 4 se observa un esquema de las causas de insuficiencia y falla intestinal.

El rango y el tipo de terapia nutricional requerida en pacientes con insuficiencia intestinal depende de la extensión y ubicación de los segmentos intestinales enfermos, principalmente en el intestino delgado, que muestran alteraciones en la capacidad de absorción. Si la presentación clínica de la insuficiencia intestinal muestra una afección grave, como el síndrome del intestino corto (SIC) con falla intestinal, la suplementación intravenosa de líquidos, electrolitos y diversos nutrientes sería un componente importante de la estrategia de tratamiento. Aunque se dispone de pruebas y pautas limitadas con respecto al momento óptimo y los métodos de implementación e interrupción de la nutrición enteral (NE) o la nutrición parenteral (NP), generalmente se recomienda intentar la reintroducción de la NE tan pronto como los pacientes se recuperen de una enfermedad intestinal grave $^{(39)}$.

\subsection{Nutrición parenteral}

Dado que los antígenos de la dieta pueden ser estimulantes importantes para el sistema inmunológico de las mucosas, el reposo intestinal con nutrición parental total (NPT) se ha considerado como una opción terapéutica en la EII con falla intestinal. El objetivo de la NPT como terapia primaria para la EII es lograr el reposo intestinal, corregir los déficits nutricionales y eliminar los estímulos mucosos antigénicos. Varios estudios han analizado el efecto de la NPT y en la década de 1980, especialmente, la NPT se utilizó para tratar a pacientes con EC de moderada a grave ${ }^{(40)}$.

Müller y colaboradores evaluaron de forma prospectiva el efecto de la NPT en 30 pacientes con EC, por lo que el $83 \%$ logró la remisión, pero la recaída fue frecuente. La cirugía podría evitarse en 25 de 30 pacientes con EC complicada con 3 semanas de NPT hospitalaria seguida de 9 semanas más de NP domiciliaria ${ }^{(41)}$. Greenberg y colaboradores compararon el efecto de la NPT, la NPP con NE de baja dosis, o la NPP con una dieta nor- 
Tabla 4. Resumen de los efectos de las intervenciones nutricionales en la enfermedad inflamatoria intestinal.

\begin{tabular}{lcccccccc}
\hline & \multicolumn{3}{c}{ Enfermedad de Crohn } & \multicolumn{3}{c}{ Colitis ulcerosa } \\
\cline { 2 - 8 } & Inducir remisión & Mantener remisión & Postop. & Inducir remisión & Mantener remisión & Postop. & Pouchitis \\
\hline NE & + & ++ & + & - & - & - & $i ?$ \\
NPT & + & - & $i ?$ & - & - & & $i ?$ \\
Probióticos & - & - & - & + & + & - & ++ \\
\hline
\end{tabular}

Postop: postoperatorio. NE: nutrición enteral, NPT: nutrición parenteral total, +: efecto positivo, -: sin efecto, ¿?: No determinado.

Tabla 5. Resumen de intervenciones nutricionales médicas en la enfermedad de Crohn activa. (Wark et al(49)).

\begin{tabular}{|c|c|c|}
\hline Intervención & Descripción & Evidencia \\
\hline Nutrición enteral exclusiva (NEE) & $\begin{array}{l}100 \% \text { de los nutrientes como fórmula líquida por } \\
\text { vía oral o por sonda nasogástrica. }\end{array}$ & $\begin{array}{l}\text { Efecto positivo pero inferior a los corticosteroides } \\
\text { para lograr la remisión. }\end{array}$ \\
\hline \multirow[t]{2}{*}{ Nutrición enteral parcial (NEP) } & $\begin{array}{l}50 \% \text { de nutrición enteral más } 50 \% \text { de dieta sin } \\
\text { restricciones. }\end{array}$ & Inferior a NEE. \\
\hline & $\begin{array}{l}50 \% \text { de nutrición enteral más } 50 \% \text { de alimentos } \\
\text { integrales selectos. }\end{array}$ & $\begin{array}{l}\text { Equivalente a NEE en tasas de respuesta } \\
\text { Mejora de la aceptabilidad en comparación con } \\
\text { NEE. }\end{array}$ \\
\hline $\begin{array}{l}\text { Dieta de alimentos comunes } \\
\text { (CD-TREAT) }\end{array}$ & $\begin{array}{l}\text { Dieta con exclusión de componentes específicos } \\
\text { en común con NEE como gluten, lactosa y } \\
\text { alcohol. }\end{array}$ & $\begin{array}{l}\text { Marcadores inflamatorios reducidos y actividad } \\
\text { de la enfermedad en la semana } 12 \text { y mejor } \\
\text { tolerada que la NEE, }\end{array}$ \\
\hline Dieta específica de carbohidratos & $\begin{array}{l}\text { Excluye granos, azúcares, alimentos procesados } \\
\text { y la mayoría de los lácteos. }\end{array}$ & Reducción de la inflamación a las 12 semanas. \\
\hline
\end{tabular}

mal suplementaria. No hubo diferencias significativas en las tasas de remisión: $71 \%$ en el grupo de NPT, $58 \%$ en el grupo de NPP con NE y $60 \%$ en el grupo de NPP y dieta normal ${ }^{(42)}$. La NPT desempeña un papel en la curación de las fístulas enterocutáneas posoperatorias que surgen de una anastomosis quirúrgica o de fístulas complicadas en la $\mathrm{EC}^{(38)}$. Cuando se comparan NPT y NE, la NPT se asocia con costos más altos y riesgos significativos, incluida la sepsis asociada al catéter venoso central, y debe restringirse a pacientes con falla intestinal que no pueden recibir una nutrición adecuada por vía ente$\mathrm{ral}^{(43)}$.

\subsection{Rol de los probióticos}

Las estrategias que modulan la disbiosis podrían ser una opción terapéutica en la EII. Los probióticos pueden mejorar el equilibrio microbiano intestinal, mejorando la función de barrera intestinal y mejorando la respuesta inmune local. Sin embargo, no existe evidencia sólida que justifique el uso de ninguna de las cepas probióticas que se han probado en el pasado en el tratamiento de la $\mathrm{EC}^{(44,45)}$. El uso de probióticos en pacientes con EC ha producido resultados ambiguos y los ensayos disponibles son pequeños, con muy pocos ensayos controlados, aleatorizados y doble ciego. Los datos más convincentes hasta ahora sobre el efecto de los probióticos en la EII se han encontrado en la prevención y el mantenimiento de la remisión de la pouchitis. Varios estudios confirman la eficacia del uso del preparado probiótico \#VSL3 para prevenir la pouchitis o mantener la remisión en pacientes en que se indujo la misma por medio de antibioticoterapia ${ }^{(46)}$.

En la tabla 4 se resumen los efectos de las intervenciones nutricionales en la enfermedad inflamatoria intestinal y en la tabla 5 se exponen las intervenciones nutricionales en la enfermedad de Crohn. Mientras que en la tabla 6 se observa un esquema para guiar el tratamiento nutricional en pacientes con enfermedad activa complicada.

\section{Conclusiones}

La terapia farmacológica sigue siendo el pilar del tratamiento de la EII. Sin embargo, los aspectos nutricionales son especialmente relevantes ya que potencialmente 
Tabla 6. Tratamiento nutricional (TN) en pacientes con Ell. Consideraciones actuales sobre nutrientes específicos y micronutrientes aplicables a pacientes con Ell (adaptado de Bischoff et al (50)).

\begin{tabular}{|c|c|c|}
\hline \multicolumn{3}{|l|}{ Macronutrientes } \\
\hline Energía & \multicolumn{2}{|c|}{ Similares a los de la población sana (30-35 kcal/kg/día) } \\
\hline Proteínas & \multicolumn{2}{|c|}{ En etapa de remisión no son elevados (1 g/kg/día) } \\
\hline & \multicolumn{2}{|c|}{ En etapa activa (brote) los requerimientos aumentan. (1,2 a 1,5 g/kg/día). } \\
\hline Lípidos & \multicolumn{2}{|c|}{$\begin{array}{l}\text { Similares a los de la población sana ( } 0,8 \text { - } 1 \mathrm{~g} / \mathrm{kg} / \mathrm{día} \text { ) Los lípidos intravenosos (incluidas las } \\
\text { fuentes de lípidos no nutricionales) no deben exceder } 1 \mathrm{~g} / \mathrm{kg} / \mathrm{dí} \text { día. } \\
\text { En NP, considerar aporte de emulsiones lipídicas de alternativa (ahorradoras de soja). }\end{array}$} \\
\hline \multicolumn{3}{|l|}{ Micronutrientes } \\
\hline Nutriente & Requerimientos diarios. & $\begin{array}{l}\text { Reemplazo recomendado en caso de } \\
\text { deficiencias (dosis oral) * }\end{array}$ \\
\hline Zinc & $15 \mathrm{mg}$ & $50 \mathrm{mg}$ elemental/día \\
\hline Hierro & $10-15 \mathrm{mg}$ & $\begin{array}{c}\mathrm{Hb}(\mathrm{g} / \mathrm{L}): \\
100-130: 1.000-1.500 \mathrm{mg} \\
70-100: 1.500-2.000 \mathrm{mg} \\
\text { El hierro oral debe considerarse como } \\
\text { tratamiento de primera línea en pacientes con } \\
\text { anemia leve. } \\
\text { Hierro intravenoso en pacientes con Ell activa } \\
\text { y/o hemoglobina < de } 100 \mathrm{~g} / \mathrm{L} .\end{array}$ \\
\hline Vitamina B 12 & $3 \mu \mathrm{g}$ & $1000 \mu \mathrm{g} / \mathrm{dí}$ \\
\hline Folato & $400 \mu \mathrm{g}$ & $1 \mathrm{mg} / \mathrm{di} a$ \\
\hline Calcio & $800-1.500 \mathrm{mg}$ & $1.500-2.000 \mathrm{mg} / \mathrm{dí} a$ \\
\hline Magnesio & $400 \mathrm{mg}$ & $150 \mathrm{mg}$ de Mg elemental 4 veces por día \\
\hline Vitamina D & $400 \mathrm{UI}$ & Dosis variable \\
\hline \multicolumn{3}{|l|}{ Recomendaciones para pacientes con Ell complicada. } \\
\hline Pacientes con EC en tratamiento con colestiramina & \multicolumn{2}{|c|}{$\begin{array}{l}\text { Riesgo adicional mínimo de malabsorción de grasas. } \\
\text { No necesitan diferencias en el TN en comparación con otros pacientes con EC. }\end{array}$} \\
\hline Pacientes con Ell con hiperoxaluria & \multicolumn{2}{|c|}{$\begin{array}{l}\text { Frecuentemente presentan malabsorción de grasas. } \\
\text { Estos pacientes deben recibir asesoramiento sobre malabsorción de grasas. }\end{array}$} \\
\hline $\begin{array}{l}\text { Pacientes con Ell con diarrea o yeyunostomía } \\
\text { de alto débito }\end{array}$ & \multicolumn{2}{|c|}{$\begin{array}{l}\text { Se debe monitorizar diuresis y electrolitos en plasma y orina. } \\
\text { El aporte parenteral o ingesta de líquidos debe adaptarse. } \\
\text { Reducir líquidos hipotónicos y aumentar soluciones salinas. }\end{array}$} \\
\hline Pacientes con estenosis y/o síntomas obstructivos. & \multicolumn{2}{|c|}{$\begin{array}{l}\text { Se puede recomendar un régimen con textura adaptada o NE distal a la estenosis } \\
\text { (postestenosis) }\end{array}$} \\
\hline
\end{tabular}

* Las cantidades son pautas generales y deben ajustarse según las necesidades individuales de cada paciente. EII: enfermedad inflamatoria intestinal; NE: nutrición enteral.

influyen en la actividad de la enfermedad y, por tanto, en su morbilidad. La disbiosis inducida por la nutrición contribuye a la patogenia de la EII, por lo que las modificaciones en la dieta tanto para la prevención como para el tratamiento de la EII son una estrategia de tratamiento deseable. Parece sustancial que los médicos clí- nicos conozcan los componentes específicos de la dieta que ejercen efectos tanto proinflamatorios como antiinflamatorios en el contexto de EC y CU. Probablemente la terapia nutricional encuentre su lugar en un enfoque de tratamiento personalizado de estos pacientes en la esfera de una estrategia de tratamiento necesariamente 
multidisciplinario que permita lograr un control óptimo de la enfermedad y minimizar los efectos secundarios no deseados.

\section{Abstract}

There is vast epidemiological evidence based on cohort studies of large populations of humans, that diet exerts a strong influence on the development and outcome of inflammatory bowel disease. Besides, most evidence-based medical therapies for this condition aim to suppress the immune response and entail significant side effects. Current evidence has proved that several diet factors probably protect against outbreaks, as in the case of fibre, zinc and vitamin D in both forms of inflammatory bowel disease and a high proportion of polyunsaturated fatty acids (PUFAs) $n-3 / n-6$ in the ICU diet. Other foods also impact the outbreak risk, such as those containing myristic acid, red meats and sugar. This review will explore diet interactions and the immune system within the context of Crohn's disease and ulcerative colitis, focusing on the role of diet in the pathogenesis, in particular in terms of its interaction with the patient's immune system. Simultaneously, the role of medical nutrition will be analysed as a therapeutic intervention.

\section{Resumo}

Há evidências epidemiológicas em seres humanos de estudos de coorte de grandes populações que a dieta tem uma forte influência no desenvolvimento e no curso da Doença Inflamatória Intestinal (DII). Além disso, a maioria dos tratamentos médicos baseados em evidências para DII têm como objetivo suprimir a resposta imunológica e acarretam riscos de efeitos colaterais significativos. A evidência atual mostrou vários fatores dietéticos que provavelmente protegem contra surtos, como fibra, zinco e vitamina $\mathrm{D}$ em ambas as formas de DII e uma alta proporção de ácidos graxos poliinsaturados (PUFAs) $n-3 / n-6$ na dieta na colite ulcerativa (UC). Outros fatores que aumentam o risco de surtos são os alimentos que contêm ácido mirístico, a carne vermelha e o açúcar. Esta revisão explora as interações da dieta e do sistema imunológico no contexto da Doença de Crohn (DC) e da Colite Ulcerativa (UC), com foco no papel da dieta na patogênese, em particular na interação com o sistema imunológico do paciente. Ao mesmo tempo, faz-se uma análise do papel da nutrição médica como intervenção terapêutica.

\section{Bibliografía}

1. Forbes A, Escher J, Hébuterne X, Klek S, Krznaric Z, Schneider S, et al. ESPEN guideline: clinical nutrition in inflammatory bowel disease. Clin Nutr 2017; 36(2):321-47. doi: 10.1016/j.clnu.2016.12.027.
2. Sartor RB, Wu GD. Roles for intestinal bacteria, viruses, and fungi in pathogenesis of inflammatory bowel diseases and therapeutic approaches. Gastroenterology 2017; 152(2):327-39.e4. doi: 10.1053/j.gastro.2016.10.012.

3. Kaplan GG, Ng SC. Understanding and preventing the global increase of inflammatory bowel disease. Gastroenterology 2017; 152:313-21. doi: 10.1053/j.gastro.2016.10.020.

4. Magro F, Gionchetti P, Eliakim R, Ardizzone S, Armuzzi A, Barreiro-de Acosta M, et al. Third european evidence-based consensus on diagnosis and management of ulcerative colitis. J Crohns Colitis 2017; 11(6):649-70. doi: 10.1093/ecco-jcc/jjx008.

5. Baumgart DC, Sandborn WJ. Inflammatory bowel disease: clinical aspects and established and evolving therapies. Lancet 2007; 369(9573):1641-57. doi: 10.1016/S0140-36(07) 60751-X.

6. Celiberto LS, Graef FA, Healey GR, Bosman ES, Jacobson K, Sly LM, et al. Inflammatory bowel disease and immunonutrition: novel therapeutic approaches through modulation of diet and the gut microbiome. Immunology 2018; 155:36-52. doi: 10.1111/imm. 12939 .

7. Yamamoto T, Nakahigashi M, Saniabadi AR. Review article: diet and inflammatory bowel disease-epidemiology and treatment. Aliment Pharmacol Ther 2009; 30(2):99-112. doi: 10.1111/j.1365-2036.2009.04035.x.

8. Ryan E, McNicholas D, Creavin B, Kelly ME, Walsh T, Beddy D. Sarcopenia, and inflammatory bowel disease: a systematic review. Inflamm Bowel Dis 2019; 25:67-73. doi: 10.1093/ibd/izy212.

9. Pedersen M, Cromwell J, Nau P. Sarcopenia is a predictor of surgical morbidity in inflammatory bowel disease. Inflamm Bowel Dis 2017; 23(10):1867-72. doi 10.1097/MIB.0000000000001166.

10. Shenkin A. Micronutrients in health and disease. Postgrad Med J 2006; 82:559-67. doi: 10.1136/pgmj.2006.047670.

11. Hosomi K, Kunisawa J. The specific roles of vitamins in the regulation of immunosurveillance and maintenance of immunologic homeostasis in the gut. Immune Netw 2017; 17(1):13-9. doi: 10.4110/in.2017.17.1.13.

12. Tian T, Wang Z, Zhang J. Pathomechanisms of oxidative stress in inflammatory bowel disease and potential antioxidant therapies. Oxid Med Cell Longev 2017; 2017:4535194. doi: $10.1155 / 2017 / 4535194$.

13. Franzosa EA, Sirota-Madi A, Avila-Pacheco J, Fornelos $\mathbf{N}$, Haiser HJ, Reinker S, et al. Gut microbiome structure and metabolic activity in inflammatory bowel disease. Nat Microbiol 2019; 4(2):293-305. doi: 10.1038/s41564-0180306-4.

14. Levine A, Sigall Boneh R, Wine E. Evolving role of diet in the pathogenesis and treatment of inflammatory bowel diseases. Gut 2018; 67:1726-38. doi: 10.1136/gutjnl-2017315866 .

15. Klement E, Cohen RV, Boxman J, Joseph A, Reif S. Breastfeeding and risk of inflammatory bowel disease: a 
systematic review with meta-analysis. Am J Clin Nutr 2004; 80(5):1342-52. doi: 10.1093/ajcn/80.5.1342.

16. IBD in EPIC Study Investigators; Tjonneland A, Overvad K, Bergmann MM, Nagel G, Linseisen J, Hallmans G, et al. Linoleic acid, a dietary n-6 polyunsaturated fatty acid, and aetiology of ulcerative colitis: a nested case-control study within a European prospective cohort study. Gut 2009; 58:1606-11. doi: 10.1136/gut.2008.169078.

17. Torki M, Gholamrezaei A, Mirbagher L, Danesh M, Kheiri S, Emami MH. Vitamin D deficiency associated with disease activity in patients with inflammatory bowel diseases. Dig Dis Sci 2015; 60(10):3085-91.

18. Kim SE. Importance of nutritional therapy in the management of intestinal diseases: beyond energy and nutrient supply. Intest Res 2019; 17(4):443-54. doi: 10.5217/ir. 2019.00075 .

19. Lewis JD, Albenberg L, Lee D, Kratz M, Gottlieb K, Reinisch W. The importance and challenges of dietary intervention trials for inflammatory bowel disease. Inflamm Bowel Dis 2017; 23(2):181-91.

20. Sigall-Boneh R, Levine A, Lomer M, Wierdsma N, Allan $\mathbf{P}$, Fiorino G, et al. Research gaps in diet and nutrition in inflammatory bowel disease: a topical review by D-ECCO Working Group [Dietitians of ECCO] J Crohns Colitis 2017; 11(12):1407-19. doi: 10.1093/ecco-jcc/jjx109.

21. Gatti S, Galeazzi T, Franceschini E, Annibali R, Albano V, Verma AK, et al. Effects of the exclusive enteral nutrition on the microbiota profile of patients with Crohn's Disease: a systematic review. Nutrients 2017; 9:832. doi: 10.3390/nu9080832.

22. Fernández-Banares F, Cabré E, Esteve-Comas M, Gassull MA. How effective is enteral nutrition in inducing clinical remission in active Crohn's disease? A meta-analysis of the randomized clinical trials. JPEN J Parenter Enteral Nutr $1995 ; 19: 356-64$

23. Zachos M, Tondeur M, Griffiths AM. Enteral nutritional therapy for induction of remission in Crohn's disease. Cochrane Database Syst Rev 2007; (1):CD000542.

24. Guagnozzi D, González-Castillo S, Olveira A, Lucendo AJ. Nutritional treatment in inflammatory bowel disease. An update. Rev Esp Enferm Dig 2012; 104:479-88. doi: 10.4321/S1130-01082012000900006.

25. Narula N, Dhillon A, Zhang D, Sherlock ME, Tondeur M, Zachos M. Enteral nutritional therapy for induction of remission in Crohn's disease. Cochrane Database Syst Rev 2018; 4(4):CD000542. doi: 10.1002/14651858.CD000542.pub3.

26. Tsertsvadze A, Gurung T, Court R, Clarke A, Sutcliffe P. Clinical effectiveness and cost-effectiveness of elemental nutrition for the maintenance of remission in Crohn's disease: a systematic review and meta-analysis. Health Technol Assess 2015; 19(26):1-138. doi: 10.3310/hta19260.

27. Wall C, Gearry R, Day A. Treatment of active Crohn's disease with exclusive and partial enteral nutrition: a pilot study in adults. Inflamm Intest Dis 2018; 2:219-27. doi: 10.1159/000489630.
28. Li Y, Zuo L, Zhu W, Gong J, Zhang W, Gu L, et al. Role of exclusive enteral nutrition in the preoperative optimization of patients with Crohn's disease following immunosuppressive therapy. Medicine (Baltimore) 2015; 94(5):e478.

29. Yamamoto T, Nakahigashi M, Umegae S, Matsumoto K. Prospective clinical trial: enteral nutrition during maintenance infliximab in Crohn's disease. J Gastroenterol 2010; 45:24-9.

30. Hirakawa H, Fukuda Y, Tanida N, Hosomi M, Shimoyama T. Home elemental enteral hyperalimentation (HEEH) for the maintenance of remission in patients with Crohn's disease. Gastroenterol Jpn 1993; 28:379-84

31. Koga H, Iida M, Aoyagi K, Matsui T, Fujishima M. [Long-term efficacy of low residue diet for the maintenance of remission in patients with Crohn's disease] Nihon Shokakibyo Gakkai Zasshi 1993; 90(11):2882-8.

32. Takagi S, Utsunomiya K, Kuriyama S, Yokoyama H, Takahashi S, Iwabuchi M, et al. Effectiveness of an 'half elemental diet' as maintenance therapy for Crohn's disease: a randomized-controlled trial. Aliment Pharmacol Ther 2006; 24:1333-40.

33. Hanai H, Iida T, Takeuchi K, Arai H, Arai O, Abe J, et al. Nutritional therapy versus 6-mercaptopurine as maintenance therapy in patients with Crohn's disease. Dig Liver Dis 2012; 44:649-54

34. Yamamoto T, Nakahigashi M, Saniabadi AR, Iwata T, Maruyama Y, Umegae S, et al. Impacts of long-term enteral nutrition on clinical and endoscopic disease activities and mucosal cytokines during remission in patients with Crohn's disease: a prospective study. Inflamm Bowel Dis 2007; 13(12):1493-01. doi: 10.1002/ibd.20238.

35. Johnson T, Macdonald S, Hill SM, Thomas A, Murphy MS. Treatment of active Crohn's disease in children using partial enteral nutrition with liquid formula: a randomised controlled trial. Gut 2005; 55:356-61. doi: 10.1136/ gut.2004.062554.

36. Levine A, Wine E, Assa A, Sigall Boneh R, Shaoul R, Kori M, et al. Crohn's disease exclusion diet plus partial enteral nutrition induces sustained remission in a randomised controlled trial. Gastroenteroly 2019; 157:440-50.e8. doi: 10.1053/j.gastro.2019.04.021.

37. Svolos V, Hansen R, Nichols B, Quince C, Ijaz UZ, Papadopoulou RT, et al. Treatment of active Crohn's disease with an ordinary food-based diet that replicates exclusive enteral nutrition. Gastroenterology 2019; 156:1354-67. doi: 10.1053/j.gastro.2018.12.002.

38. Kim SE. Importance of nutritional therapy in the management of intestinal diseases: beyond energy and nutrient supply. Intest Res 2019;17(4):443-54. doi: 10.5217/ir. 2019.00075 .

39. Albenberg LG, Lewis JD, Wu GD. Food, and the gut microbiota in inflammatory bowel diseases: a critical connection. Curr Opin Gastroenterol 2012; 28:314-20. 
40. Müller JM, Keller HW, Erasmi H, Pichlmaier H. Total parenteral nutrition as the sole therapy in Crohn's disease- - a prospective study. Br J Surg 1983; 70(1):40-3.

41. Greenberg GR, Fleming CR, Jeejeebhoy KN, Rosenberg IH, Sales D, Tremaine WJ. Controlled trial of bowel rest and nutritional support in the management of Crohn's disease. Gut 1988; 29:1309-15.

42. Pironi L, Arends J, Bozzetti F, Cuerda C, Gillanders L, Jeppesen PB, et al. ESPEN guidelines on chronic intestinal failure in adults. Clin Nutr 2016; 35(2):247-307. doi: 10.1016/j.clnu.2016.01.020.

43. Richman E, Rhodes JM. Review article: evidence-based dietary advice for patients with inflammatory bowel disease. Aliment Pharmacol Ther 2013; 38:1156-71.

44. Rolfe VE, Fortun PJ, Hawkey CJ, Bath-Hextall F. Probiotics for maintenance of remission in Crohn's disease. Cochrane Database Syst Rev 2006; (4):CD004826. doi: 10.1002/14651858.CD004826.pub2.

45. Butterworth AD, Thomas AG, Akobeng AK. Probiotics for induction of remission in Crohn's disease. Cochrane Database Syst Rev 2008; (3):CD006634. doi: 10.1002/ 14651858.CD006634.pub2.

46. Magro F, Gionchetti P, Eliakim R, Ardizzone S, Armuzzi A, Barreiro-de Acosta M, et al. Third european eviden- ce-based consensus on diagnosis and management of ulcerative colitis. Part 1: Definitions, diagnosis, extra-intestinal manifestations, pregnancy, cancer surveillance, surgery, and ileo-anal pouch disorders. J Crohns Colitis 2017; 11(6): 649-70.

47. Balestrieri P, Ribolsi M, Guarino MPL, Emerenziani S, Altomare A, Cicala M. Nutritional aspects in inflammatory bowel diseases. Nutrients 2020; 12(2):372. doi: 10.3390/nu12020372.

48. Kilby K, Mathias H, Boisvenue L, Heisler C, Jones JL. Micronutrient absorption and related outcomes in people with inflammatory bowel disease: a review. Nutrients 2019; 11(6):1388. doi: 10.3390/nu11061388.

49. Wark G, Samocha-Bonet D, Ghaly S, Danta M. The role of diet in the pathogenesis and management of inflammatory bowel disease: a review. Nutrients 2020; 13(1):135. doi: 10.3390/nu13010135.

50. Bischoff SC, Escher J, Hébuterne X, Klêk S, Krznaric Z, Schneider S, et al. ESPEN practical guideline: clinical nutrition in inflammatory bowel disease. Clin Nutr 2020; 39(3):632-53. doi: 10.1016/j.clnu.2019.11.002.

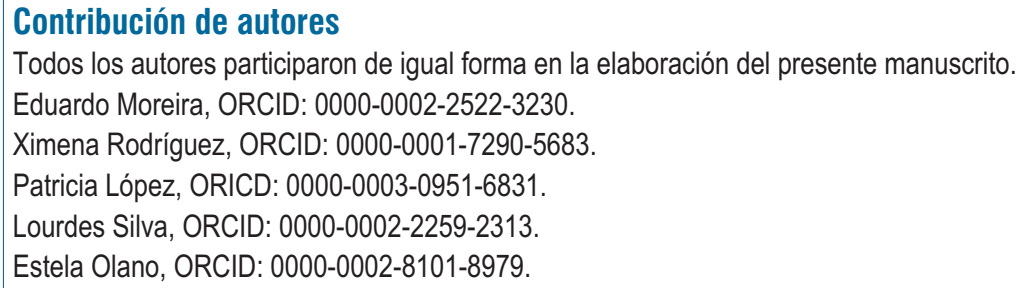

